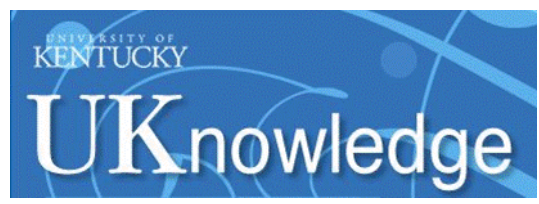

University of Kentucky

UKnowledge

5-16-2013

\title{
Studies of NGC 6720 with Calibrated HST/WFC3 Emission-line Filter Images. III. Tangential Motions using AstroDrizzle Images
}

C. R. O'Dell

Vanderbilt University

Gary J. Ferland

University of Kentucky, gary@uky.edu

W. J. Henney

Universidad Nacional Autónoma de México, Mexico

M. Peimbert

Universidad Nacional Autónoma de México, Mexico

Follow this and additional works at: https://uknowledge.uky.edu/physastron_facpub

Part of the Astrophysics and Astronomy Commons, and the Physics Commons

Right click to open a feedback form in a new tab to let us know how this document benefits you.

\section{Repository Citation}

O'Dell, C. R.; Ferland, Gary J.; Henney, W. J.; and Peimbert, M., "Studies of NGC 6720 with Calibrated HST/WFC3 Emission-line Filter Images. III. Tangential Motions using AstroDrizzle Images" (2013). Physics and Astronomy Faculty Publications. 60.

https://uknowledge.uky.edu/physastron_facpub/60

This Article is brought to you for free and open access by the Physics and Astronomy at UKnowledge. It has been accepted for inclusion in Physics and Astronomy Faculty Publications by an authorized administrator of UKnowledge. For more information, please contact UKnowledge@lsv.uky.edu. 
Studies of NGC 6720 with Calibrated HST/WFC3 Emission-line Filter Images. III. Tangential Motions using AstroDrizzle Images

Digital Object Identifier (DOI)

https://doi.org/10.1088/0004-6256/145/6/170

Notes/Citation Information

Published in The Astronomical Journal, v. 145, no. 6, 170, p. 1-8.

(C) 2013. The American Astronomical Society. All rights reserved. Printed in the U.S.A.

The copyright holder has granted permission for posting the article here. 


\title{
STUDIES OF NGC 6720 WITH CALIBRATED HST/WFC3 EMISSION-LINE FILTER IMAGES. III. TANGENTIAL MOTIONS USING ASTRODRIZZLE IMAGES*
}

\author{
C. R. O’Dell ${ }^{1}$, G. J. Ferland ${ }^{2}$, W. J. Henney ${ }^{3}$, and M. Peimbert ${ }^{4}$ \\ ${ }^{1}$ Department of Physics and Astronomy, Vanderbilt University, Box 1807-B, Nashville, TN 37235, USA; cr.odell@vanderbilt.edu \\ 2 Department of Physics and Astronomy, University of Kentucky, Lexington, KY 40506, USA \\ ${ }^{3}$ Centro de Radioastronomía y Astrofísica, Universidad Nacional Autónoma de México, Apartado Postal 3-72, 58090 Morelia, Michaoacán, Mexico \\ ${ }^{4}$ Instituto de Astronomia, Universidad Nacional Autónoma de México, Apdo, Postal 70-264, 04510 México D.F., Mexico \\ Received 2013 March 22; accepted 2013 April 17; published 2013 May 16
}

\begin{abstract}
We have been able to compare with astrometric precision AstroDrizzle processed images of NGC 6720 (the Ring Nebula) made using two cameras on the Hubble Space Telescope. The time difference of the observations was $12.925 \mathrm{yr}$. This large time base allowed the determination of tangential velocities of features within this classic planetary nebula. Individual features were measured in [N $\mathrm{II}]$ images as were the dark knots seen in silhouette against background nebular [O III] emission. An image magnification and matching technique was also used to test the accuracy of the usual assumption of homologous expansion. We found that homologous expansion does apply, but the rate of expansion is greater along the major axis of the nebula, which is intrinsically larger than the minor axis. We find that the dark knots expand more slowly than the nebular gas, that the distance to the nebula is $720 \mathrm{pc}$ $\pm 30 \%$, and that the dynamic age of the Ring Nebula is about $4000 \mathrm{yr}$. The dynamic age is in agreement with the position of the central star on theoretical curves for stars collapsing from the peak of the asymptotic giant branch to being white dwarfs.
\end{abstract}

Key words: instrumentation: miscellaneous - planetary nebulae: individual (Ring Nebula, NGC 6720)

\section{BACKGROUND AND INTRODUCTION}

Using the spatial motion of the material in planetary nebulae (PNe) to understand their three-dimensional (3D) structure and evolutionary processes has been a goal of many studies of these ubiquitous objects. Wilson (1950) first established the utility of radial velocity maps of a $\mathrm{PN}$ for determining its basic structure, showing that most objects could be explained as expanding ellipsoidal shells. Numerous studies since then have exploited this approach as high-velocity resolution spectrographs became more common and as high quantum efficiency CCD detectors replaced photograph emulsions. We now understand how the apparent form of many $\mathrm{PNe}$ is the result of interacting stellar winds. Slowly moving gas, expelled as the central star reaches the top of the asymptotic giant branch, is overtaken by a fast wind of gas, expelled as the star begins to heat up. This is when the central star begins its collapse toward becoming a white dwarf. In the earliest phase, the ejected material is mostly neutral and has a small ionized core. The shell may become fully ionized as the central star increases in temperature and remains at high bolometric luminosity. As the luminosity of the central star in ionizing photons drops, the outer parts of the material may again become neutral. From that point on, the balance of ionized and neutral gas becomes a subtle balance. The decreasing gas density that results from the nebula's expansion causes the ionized fraction to increase. However, the decreasing luminosity of the star causes the ionized fraction to decrease. These factors mean that the dynamic structure that we see today is the result of multiple processes, rather than the simple ejection of a shell from a collapsing star. Nevertheless, it is worthwhile studying the dynamics of the PNe because it allows us to infer what must have been their earlier conditions and to predict their futures.

\footnotetext{
* Based on observations with the NASA/ESA Hubble Space Telescope, obtained at the Space Telescope Science Institute, which is operated by the Association of Universities for Research in Astronomy, Inc., under NASA contract No. NAS 5-26555.
}

\subsection{Three-dimensional Structures from Radial Velocities}

Constraining determination of the 3D structure and dynamics only to the use of line-of-sight (radial) velocities is an important limitation. Interpretation of the observed radial velocities usually demands a simplifying assumption about the general nature of the motion. Most often the assumption is of homologous expansion; i.e., the spatial velocity away from the central star scales with the spatial distance. Homologous expansion is like the explosive expulsion of particles of varying velocity. Knowing now that the expanding material is shaped and accelerated by multiple processes makes it surprising that PNe resemble homologous expansion, a result going back to Wilson's (1950) early study. Wilson showed that emission originating farther from the central star shows a higher velocity of expansion. Mapping the radial velocities of PNe has become straightforward and common, but these data only map one component of the spatial motion $\left(V_{R}\right)$. However, one cannot underestimate the importance of Wilson's (1950) study since quantitative efforts to determine the 3D structure of PNe originate with this paper.

\subsection{Determining Tangential Velocities in Planetary Nebulae}

Potentially, one can create better models for the PNe if we also have measurements of their tangential motions. However, for a fixed value of the spatial motion perpendicular to the line of sight $\left(V_{T}\right)$, the apparent motion in the plane of the sky $(\mu)$ decreases with increasing distance $(D)$ to the target, and many fewer nearby candidate objects exist. When expressed in the common units of $V_{T}$ in $\mathrm{km} \mathrm{s}^{-1}, \mu$ in $\mathrm{yr}^{-1}$, and $D$ in parsecs, the relation is $\mu=0.211 \times V_{T} / D$. Inserting representative values of $V_{T}=20 \mathrm{~km} \mathrm{~s}^{-1}$ and $D=500 \mathrm{pc}$ gives an expected $\mu$ of $0.0089 \mathrm{yr}^{-1}, 8.9$ mas per year. Determination of $\mu$ was first attempted by Liller et al. (1966) using ground-based images of PNe obtained with several large telescopes over a period of up to 62 yr. No believable values were produced because of the blurring by astronomical seeing (characteristically about $2^{\prime \prime}$ in the older images employed) and the lack of a clear filtering of 
single emission lines. The latter limitation is because different emission lines can arise from different positions in the nebula, thus blurring the image.

As the Very Large Array (VLA) radio interferometer began to produce stable images of $1^{\prime \prime}$ resolution and better, the possibility was recognized that this telescope could provide usable $\mu$ values by special processing of the data (Masson 1986; Gómez et al. 1993; Hajian et al. 1993, 1995). A summary of results was given by Terzian (1997). Limited to the study of high surface brightness $\mathrm{PNe}$ and unable to give unambiguous results for different portions of the PNe, the method is no longer in wide use.

The VLA method of obtaining $\mu$ has essentially been succeeded by the use of two-epoch images using the Hubble Space Telescope (HST). With its stable optical-wavelength images of slightly better than $0{ }^{\prime} .1$ resolution and its ability to clearly isolate regions emitting specific emission lines, this becomes the more promising approach. Reed et al. (1999) employed pairs of images of NGC 6543 using the high-resolution CCD camera (45.5 mas pixels) of the Wide Field Planetary Camera 2 (WFPC2) with a time separation of $2.92 \mathrm{yr}$. They identified three approaches. The first approach was to measure radial profiles of samples on the two sets of images and then look for displacements using a gradient recognition algorithm. The second approach also used radial profiles, but fit Gaussian profiles to features and looked for the changes in their centers. The final technique was to magnify the first epoch images by a succession of scale factors, align their centers on the central star, and recognize at what scale factor the magnified first epoch and the second epoch images matched. This last approach utilizes all of the nebula's image, but its accuracy is difficult to quantify and works only in the case of near-homologous expansion. Motions

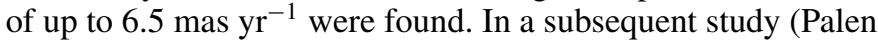
et al. 2002) of four PNe with time differences of 3.9-4.2 yr, the first and second approach were used to successfully determine $\mu$ in three objects. However, they compared broad bandpass images (the WFPC2 F555W) with monochromatic images (the WFPC2 F502N) arguing that the signal of both filters was dominated by the $\left[\mathrm{O}_{\mathrm{III}}\right]$ emission line at $500.7 \mathrm{~nm}$. Values up to

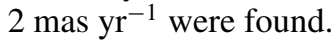

\subsection{Tangential Velocities in the Ring Nebula}

As the archetypical elliptical-form PN, NGC 6720 (henceforth the Ring Nebula) has attracted considerable attention in observational programs. However, it is significantly lower in surface brightness than most of the PNe that have been the subject of radial velocity and tangential motion studies, and highresolution spectroscopy has been a relatively recent subject. O'Dell et al. (2002) used WFPC2 [N II] emission-line images with a $1.944 \mathrm{yr}$ time difference and the magnification technique of Reed et al. (1999) to determine a magnification factor $(M)$ of $M=1.00067 \pm 0.0001 \mathrm{yr}^{-1}$. This study employed the coarser (100 mas) pixels of the wider field-of-view CCDs of the WFPC2. With an average diameter of $36^{\prime \prime}$ of its Main Ring, this

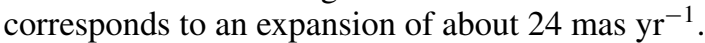

In a discussion of possibly homologous expansion it is more convenient to express the results in terms of what we call here the expansion scale factor $E$, where $E=\mu / \phi$, with $\phi$ being the angular distance from a point in the nebula to the central star. If homologous expansion applies, then $E$ will be constant. It is analogous to the Hubble constant that used to describe the expansion of the universe to the first order of accuracy. The expansion scale factor is most conveniently expressed with units of mas $\mathrm{yr}^{-1}$ arcsec. For the magnification technique, $E=(M-1) \times 10^{3}$. The O'Dell et al. (2002) results can then

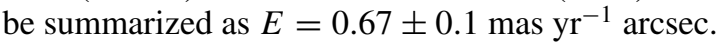

However, a study of radial velocities in the Ring Nebula (O'Dell et al. 2007) questioned the accuracy of this result because the second epoch images had the nebula centered on the central star, which meant that the image of the Main Ring fell across all four CCDs in the WFPC2, whereas the first epoch images fell onto only a single CCD. Since the relative positions of the CCDs are more difficult to determine than the astrometric corrections within a single CCD, there could be a systematic error. This limitation was circumvented in a later study (O'Dell et al. 2009) where single CCD images with a time difference of $9.557 \mathrm{yr}$ were employed. All of the techniques introduced by Reed et al. (1999) were used with the result that $E=0.23 \pm 0.1 \mathrm{mas} \mathrm{yr}^{-1}$ arcsec for [N II] emission and four dark

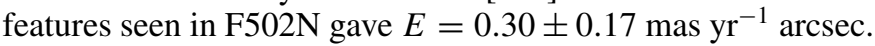
These results indicate that the O'Dell et al. (2002) numbers were inaccurate, probably because of using multiple CCDs in the second epoch images. The O'Dell et al. (2009) result also argues for the first time that the dark knots that mark the Ring Nebula and are ubiquitous in nearby PNe (O'Dell et al. 2002) expand at approximately the same rate as the nebular material. The big uncertainty in the values of $E$ means that a conclusion about commonality of motion is quite uncertain.

\subsection{Three-dimensional Modeling and the Value of Tangential Velocities in the Ring Nebula}

Attempts to model the Ring Nebula in 3D have a long history. These are summarized in a recent publication (O'Dell et al. 2013a) that draws on the extensive imaging and radial velocity mapping that has been done. In that study, it was found that the Main Ring of the nebula, where most of its optical light originates, is a non-circular disk of ionization bounded gas. Near the central axis of the disk, material extends toward the observer in the form of lobes seen only in projection onto the plane of the sky. The Main Ring is surrounded by a glow of [O III] emission and two low-ionization halos. All three of these last features are visible as fossil radiation, material that was previously photoionized but is now shielded from the ionizing radiation of the central star. Hundreds of dark knots are best seen in extinction against the Main Ring's [O III] emission with the F502N filter although many of them have low-ionization arcs on the side of the knot that faces the central star. The dark knots generally point toward the central star, but do not have the radial symmetry of form seen in the Helix Nebula (O'Dell et al. 2005).

Since the polar axis of the Ring Nebula is pointed within about $10^{\circ}$ of the observer, the Main Ring is viewed nearly in the plane of the sky and tangential velocities can be determined. These tangential velocities can be used to understand the structure and distance of the nebula. The Ring Nebula is also a prime target for addressing the important question of the relative motion of the nebular gas and the dark knots.

As part of HST program GO 12309, high signal-to-noise ratio images were made in the strongest emission lines of the entire nebula using the Wide Field Camera 3 (WFC3) that replaced the WFPC2 during the last servicing mission. These images were used to create the 3D model discussed in O'Dell et al. (2013a). Images were also with smaller field-of-view diagnostic filters and were used to determine the physical conditions (ionization, temperatures, densities) within the nebula (O'Dell et al. 2013b). The WFC3 pipeline-processed images better sample (40 mas 


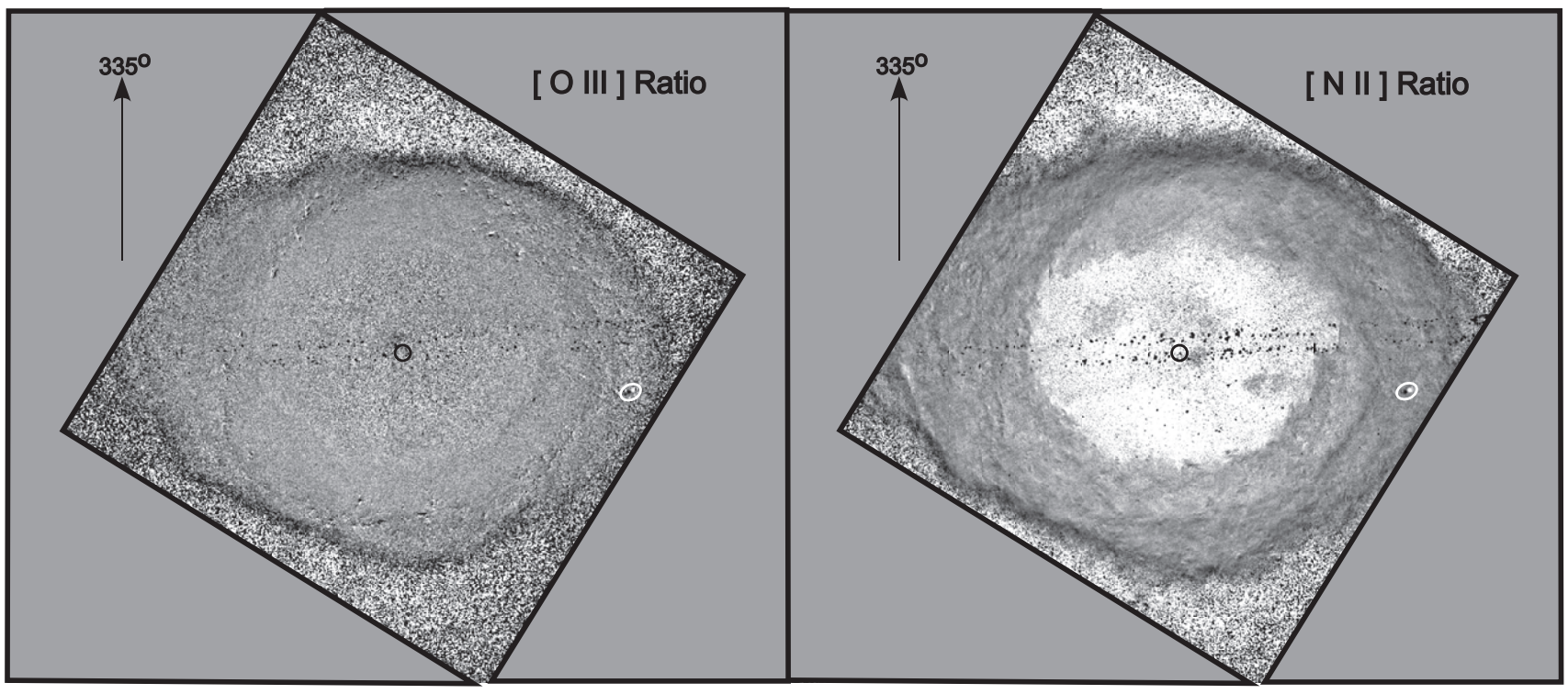

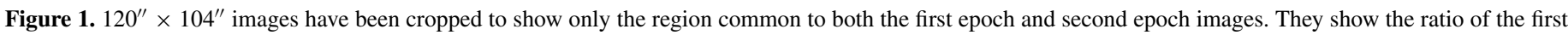

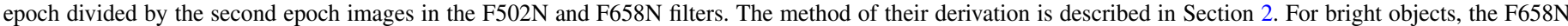

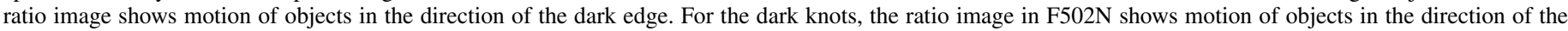

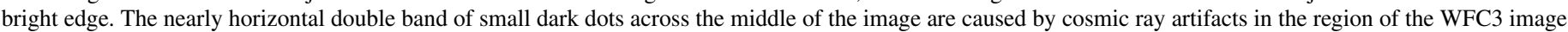

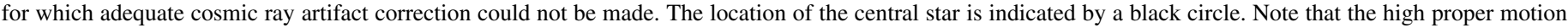
star enclosed in the white oval was not used in aligning the two-epoch images.

per pixel) the point-spread function of $H S T$ and thus provide the highest resolution images of the Ring Nebula. As a new camera, no earlier observations of the Ring Nebula exist. However, one can accurately use the earlier WFPC2 images as first epoch images because the pipeline-processed images extracted from the HST archives have had an accurate distortion correction applied, thus rendering the early and recent images geometrically "flat." The geometric correction step is called "AstroDrizzle" and is applied to both the old and new images. It is only necessary to scale the WFPC2 images to the same pixel size as the WFC3 images, align the central star images, and rotate them into alignment. One then has astrometrically useful paired images whose alignment accuracy should be about 0.2 WFC 3 pixels. Not only are these recent images of a higher spatial resolution, they offer a longer time base of $12.925 \mathrm{yr}$ when combined with the first images made in program GO 7632.

In this paper, we describe the method of aligning and measuring the image pairs (Section 2.1), present the results for measurements of gaseous features in [N II] and the dark knots in F502N (Sections 2.2 and 2.3), and discuss what this tells us about the Ring Nebula (Section 3).

\section{THE IMAGES}

The images used in this study were obtained in the HST programs GO 7632 (WFPC2, 1998 October 16) and GO 12309 (WFC3, 2011 September 19). Multiple exposures were made at each epoch, thus allowing cosmic ray event correction. The images in filter F502N well isolate the $500.7 \mathrm{~nm}$ line of [O III], and the images in filter F658N well isolate the $658.3 \mathrm{~nm}$ line of [N II]. The [N II] emission arises from a thin layer close to the main ionization front of the nebula (O'Dell et al. 2013a). In contrast, the [O III] emission arises from an extended region lying between the $[\mathrm{N} \mathrm{II}]$ emitting layer and the He II core in the center of the nebula (O'Dell et al. 2013a). This means that the F658N images provide a means of tracing the motion of gas near the main ionization front, while the primary value of the F502N images lies in their ability to show the dark knots in detail.

\subsection{Combining the WPC2 and WFC3 Images}

The first epoch GO 7632 AstroDrizzle images were downloaded from the $H S T$ data archive and put into the same pixel scale as the second epoch GO 12309 AstroDrizzled by using the IRAF" task "magnify" and the scaling factor of 2.5262758 . The scaling factor could not be determined from the few stars in the Ring Nebula images. This means that we adopted the value obtained by comparing rich star fields in the Orion Nebula Cluster imaged with the WFPC2 in program GO 5085 and with the WFC3 in program GO 12543. The two sets of images were aligned with IRAF task "geomap/rotate" using the four stars available. The alignment accuracy of the central star is about 3 mas, and the determination accuracy of the rotation is about 0.015 .

The aligned images were scaled to the same signal level, and the WFC3 image was blurred to the same stellar image point-spread function as the WFPC2 image using the IRAF task "gauss." A ratio of the first and second epoch images was made, with the second epoch as the denominator. These ratio images revealed motions that had occurred during the 12.925 yr interval between the observations. For bright objects, the F658N ratio image shows motion of objects in the direction of the dark edge. For the dark knots, the ratio image in F502N shows motion of objects in the direction of the bright edge. These images are shown in Figure 1.

\subsection{Measuring the Motion of Features with a Least-squares Method}

The ratio images were used as a guide in determining samples to be measured using a least-squares code developed by Hartigan

\footnotetext{
5 IRAF is distributed by the National Optical Astronomy Observatories, which is operated by the Association of Universities for Research in Astronomy, Inc., under cooperative agreement with the National Science
} foundation. 


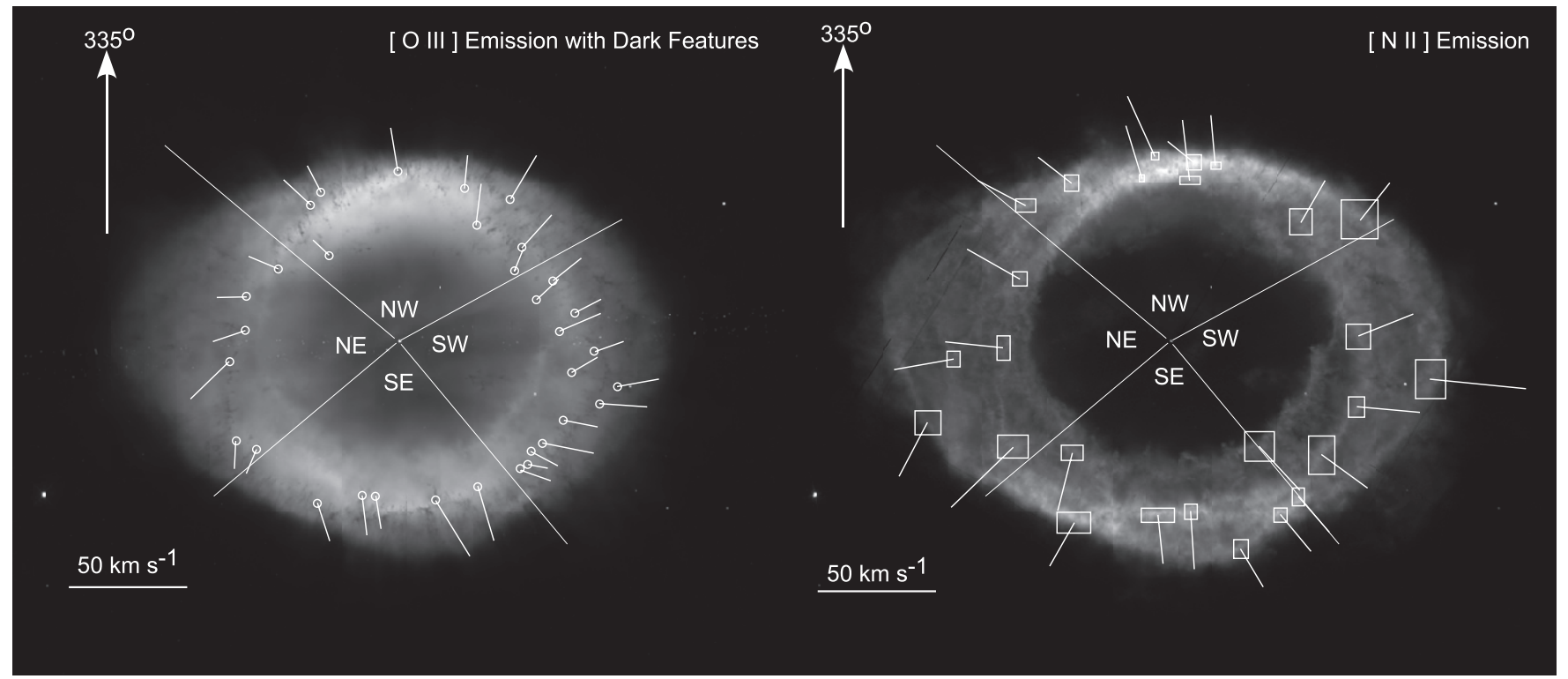

Figure 2. Same fields of view as in Figure 1 are shown. The motions determined by the ZSQ method described in Section 2 are shown as vectors. At an assumed distance of $720 \mathrm{pc}$, a motion of one ACS pixel corresponds to a tangential velocity of $10.6 \mathrm{~km} \mathrm{~s}^{-1}$. The velocity scale for the vectors is shown on both panels. The small samples used for the determination of the dark knots' motions are shown as open circles in the F502N image dominated by background [O III] emission and the larger samples used for the determination of the bright [N II] features' motions are shown as open boxes in the [N II] image.

et al. (2001) from an approach originated by Currie et al. (1996) that compares incrementally shifted images. The motion is assumed to be correct when there is a minimum difference between the reference and shifted image. In this paper we call this method the ZSQ method. The results for both small dark features seen in the F502N images and large bright features seen in the F658N images are shown in Figure 2. Results for individual determinations of $\mu$ are given in Tables 1 and 2. After recognizing patterns in the tangential velocities, we grouped the $\mu$ values into quadrants within the Main Ring as shown in Figure 2. The averages within the quadrants are presented in Table 3

It is possible to approximate the uncertainties of the measurements. The ZSQ gives much smaller uncertainties than indicated by a comparison of $\mu$ for two nearby objects. Excluding the F502N dark features in the SW quadrant (cf. Section 3), the average motion of all features is $2.12 \pm 0.33$ Advanced Camera for Surveys (ACS) pixels. Over the time interval of

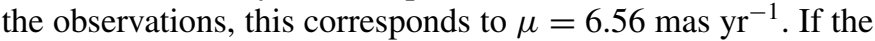
alignment uncertainty is that of the central star image (3 mas or 0.08 pixels), that component of the uncertainty of the magnitude

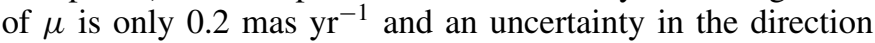
of motion of 2.2 . The average distance of this set of samples is $27^{\prime \prime} .5$ (688 pixels). The uncertainty in the rotation between the two sets of images $(0.015)$ would cause the tips of the features to be displaced by 0.18 pixels, which would produce an error of 4.9 in the direction of motion. These two considerations mean that uncertainty in the alignment propagates into small uncertainties in the derived magnitude and direction of the motion of features in the nebula. Typically, the uncertainties in the ZSQ solutions are about 0.05 pixels, which corresponds to a $3 \%$ uncertainty in the magnitude of $\mu$ and an uncertainty of about 1.5 . The significant differences in the magnitude and direction of $\mu$ for nearby objects in the Main Ring are likely to be real and can represent significant small-scale fluctuations in the gas flow of the Main Ring. An alternative explanation may be that two adjacent lines of sight are dominated by radiation from very different parts of the 3D Main Ring.

\subsection{Measuring Motions with the Magnification Method}

We adopted a variation of the magnification method of determining motions. The underlying assumption of the method is that the tangential motions are homologous. When a correctly magnified first epoch image is aligned with the second epoch image (using the central star) and a ratio of the two is made, a flat image of constant value should result. However, if the expansion scale factor is not constant in all parts of the nebula, employing a single optimum magnification (where the ratio of images becomes constant) will not fully represent what is happening. Recall from Section 1.3 that $E$ and the magnification $(M)$ are related by $E=(M-1) \times 10^{3}$ when $E$ is conveniently expressed in units of mas $\mathrm{yr}^{-1}$ arcsec. We determined the optimum magnification and $E$ for individual regions within the image of the Main Ring, as shown in Figure 3. The F502N samples were selected from where the nebular background varied little and contained numerous dark knots. The results should represent the motion of the dark knots. The F658N samples were regions of fine-scale structure in the [N II] image. The results should indicate motion of ionization fronts aligned with the observer's line of sight.

Several steps were used to derive the optimum magnification. Initially, the first and second epoch images were scaled to the same signal level. The first epoch images were magnified in steps of 0.00005 . The central star was aligned on the resultant images, and a ratio of the two images was made for each magnification. Finally, the IRAF task "imstat" was applied to establish the standard deviation (STDDEV) of the signal within the sampled region. There was always a difference between the two images. The STDDEV was largest when the magnification was furthest from optimum because the ratio image was the result of comparing two distinct images, each with its own characteristics. However, as the magnification factor reached the optimum value, the STDDEV reached a minimum, and then increased at values larger than the optimum magnification. The STDDEV was never zero because the signal-to-noise ratio of the first and second epoch images was 


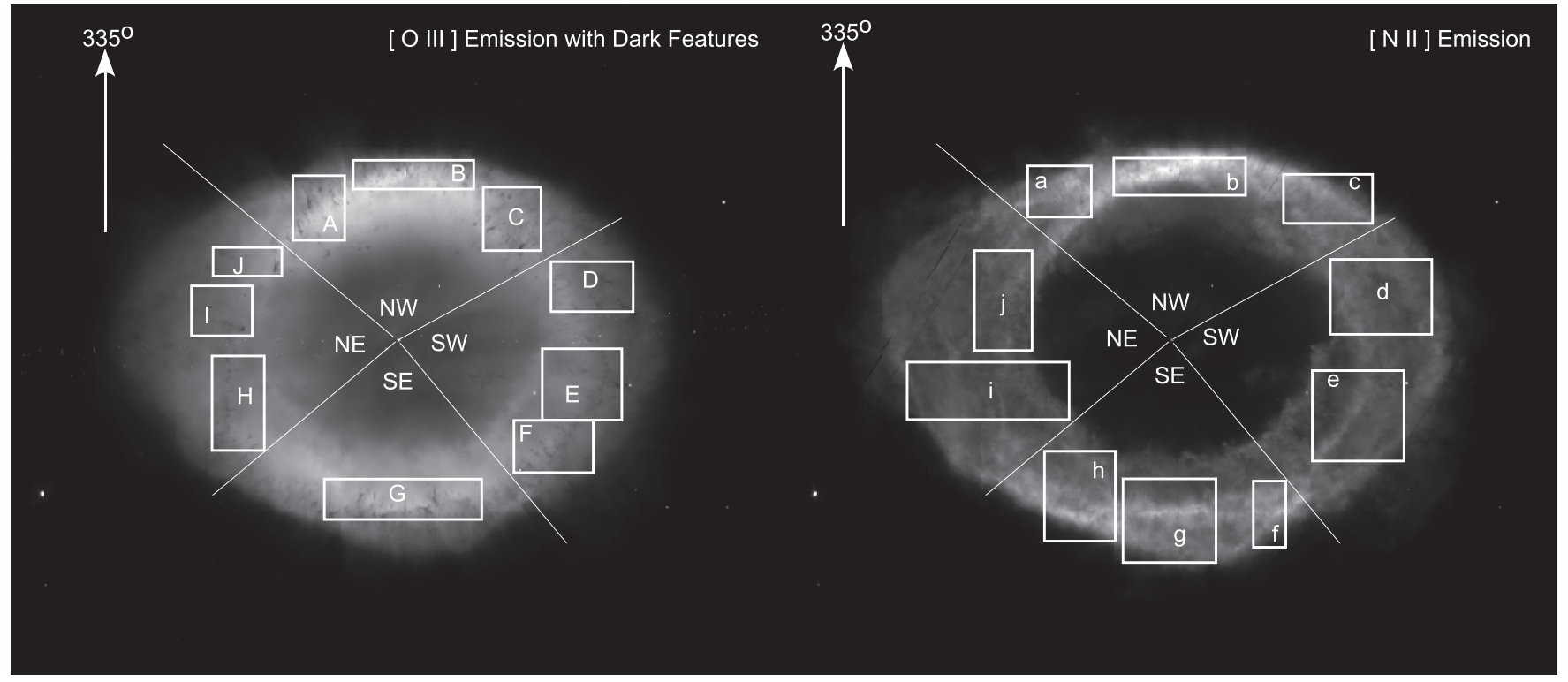

Figure 3. Same fields of view as in Figure 1 are shown. The areas used in determining the optimum magnification are designated by heavy closed rectangles.

Table 1

ZSQ Method Motions for [N II] Bright Features

\begin{tabular}{|c|c|c|c|c|c|}
\hline $\begin{array}{l}\text { Sample-P.A. }{ }^{\mathrm{a}} \\
\left({ }^{\circ}\right)\end{array}$ & $\begin{array}{c}\text { Sample-Distance } \\
\left({ }^{\prime \prime}\right)\end{array}$ & $\begin{array}{c}\mu \\
\left(\operatorname{mas~yr}^{-1}\right)\end{array}$ & $\begin{array}{c}\text { Vector-P.A. }{ }^{a} \\
\left({ }^{\circ}\right)\end{array}$ & $\begin{array}{c}E \\
\left({\text { mas } \mathrm{yr}^{-1}} \operatorname{arcsec}\right)\end{array}$ & Quadrant \\
\hline 021.9 & 30.8 & 6.51 & 037 & 0.211 & NW \\
\hline 007.1 & 28.9 & 5.11 & 027 & 0.177 & $\ldots$ \\
\hline 344.8 & 25.7 & 6.66 & 352 & 0.259 & $\ldots$ \\
\hline 339.7 & 28.8 & 7.97 & 359 & 0.277 & $\ldots$ \\
\hline 328.1 & 24.8 & 7.36 & 342 & 0.297 & $\ldots$ \\
\hline 327.1 & 27.8 & 4.14 & 026 & 0.167 & $\ldots$ \\
\hline 320.4 & 28.2 & 6.08 & 340 & 0.216 & $\ldots$ \\
\hline 287.2 & 27.4 & 5.85 & 305 & 0.213 & $\ldots$ \\
\hline 277.4 & 35.1 & 5.75 & 297 & 0.164 & $\ldots$ \\
\hline 246.5 & 29.3 & 7.12 & 267 & 0.243 & SW \\
\hline 236.5 & 40.7 & 11.63 & 239 & 0.286 & $\ldots$ \\
\hline 225.5 & 30.9 & 7.63 & 240 & 0.247 & $\ldots$ \\
\hline 208.0 & 29.4 & 6.88 & 209 & 0.234 & $\ldots$ \\
\hline 195.1 & 21.5 & 7.25 & 198 & 0.338 & $\ldots$ \\
\hline 194.3 & 31.5 & 5.71 & 196 & 0.181 & SE \\
\hline 187.3 & 31.8 & 5.79 & 195 & 0.182 & $\ldots$ \\
\hline 173.5 & 34.1 & 5.35 & 186 & 0.157 & $\ldots$ \\
\hline 161.8 & 26.7 & 7.01 & 159 & 0.263 & $\ldots$ \\
\hline 150.9 & 27.2 & 5.91 & 161 & 0.217 & $\ldots$ \\
\hline 127.3 & 32.0 & 6.03 & 126 & 0.189 & $\ldots$ \\
\hline 114.0 & 23.2 & 7.17 & 141 & 0.309 & $\ldots$ \\
\hline 099.0 & 29.5 & 10.41 & 109 & 0.353 & $\mathrm{NE}$ \\
\hline 083.5 & 39.9 & 7.34 & 127 & 0.184 & $\ldots$ \\
\hline 069.9 & 33.9 & 7.29 & 075 & 0.215 & $\ldots$ \\
\hline 067.3 & 26.1 & 7.00 & 059 & 0.268 & $\ldots$ \\
\hline 042.5 & 25.3 & 7.33 & 035 & 0.290 & $\ldots$ \\
\hline
\end{tabular}

Note. ${ }^{a}$ P.A. means the position angle, measured counterclockwise from north.

not the same. Imperfect matching of the image resolution would also contribute to a non-zero STDDEV value. The images had been approximately matched to the same point-spread function, but subtle differences in fine structure would exist and contribute to a non-zero STDDEV even at the optimum magnification. The results of the optimum magnification and the expansion scale factor determinations are given in Tables 4 and 5. The group averages of the magnification results are given in Table 6.

\section{DISCUSSION}

\subsection{Large-scale Properties of the Ring Nebula's Main Ring}

The results of the analysis of the motions were obtained in terms of average values of $E$. The quadrants we adopt are primarily meant to recognize regions of different motion, but they also agree with the natural divisions suggested by the most current 3D model (O'Dell et al. 2013a). A remarkable symmetry of features appears in opposite quadrants, which 
Table 2

ZSQ Method Motions for F502N Dark Features

\begin{tabular}{|c|c|c|c|c|c|}
\hline $\begin{array}{l}\text { Sample-P.A. }{ }^{a} \\
\left({ }^{\circ}\right)\end{array}$ & $\begin{array}{c}\text { Sample-Distance } \\
\left({ }^{\prime \prime}\right)\end{array}$ & $\begin{array}{c}\mu \\
\left(\operatorname{mas}_{\mathrm{yr}}^{-1}\right)\end{array}$ & $\begin{array}{c}\text { Vector-P.A. }{ }^{\mathrm{a}} \\
\left({ }^{\circ}\right)\end{array}$ & $\begin{array}{c}E \\
\left(\operatorname{mas} \mathrm{yr}^{-1} \operatorname{arcsec}\right)\end{array}$ & Quadrant \\
\hline 014.4 & 16.3 & 5.45 & 045 & 0.334 & NW \\
\hline 007.8 & 24.2 & 7.16 & 040 & 0.295 & $\ldots$ \\
\hline 002.4 & 25.5 & 5.86 & 032 & 0.230 & $\ldots$ \\
\hline 334.3 & 25.8 & 6.63 & 013 & 0.257 & $\ldots$ \\
\hline 310.0 & 25.4 & 4.92 & 010 & 0.194 & $\ldots$ \\
\hline 300.9 & 21.8 & 5.63 & 002 & 0.258 & $\ldots$ \\
\hline 296.7 & 28.0 & 5.23 & 336 & 0.188 & $\ldots$ \\
\hline 280.1 & 24.1 & 4.01 & 329 & 0.166 & $\ldots$ \\
\hline 274.1 & 21.1 & 3.40 & 013 & 0.161 & $\ldots$ \\
\hline 266.3 & 25.7 & 2.73 & 331 & 0.089 & SW \\
\hline 261.6 & 22.4 & 2.54 & 347 & 0.113 & $\ldots$ \\
\hline 252.6 & 28.1 & 1.64 & 247 & 0.058 & $\ldots$ \\
\hline 248.4 & 25.1 & 2.81 & 296 & 0.112 & $\ldots$ \\
\hline 240.0 & 30.6 & 1.26 & 323 & 0.041 & $\ldots$ \\
\hline 233.6 & 28.0 & 1.80 & 336 & 0.064 & $\ldots$ \\
\hline 233.1 & 34.8 & 2.05 & 271 & 0.059 & $\ldots$ \\
\hline 227.6 & 32.8 & 2.61 & 237 & 0.080 & $\ldots$ \\
\hline 219.2 & 28.6 & 1.18 & 202 & 0.041 & $\ldots$ \\
\hline 209.6 & 27.5 & 3.28 & 222 & 0.119 & $\ldots$ \\
\hline 205.5 & 26.7 & 1.76 & 169 & 0.066 & $\ldots$ \\
\hline 201.3 & 27.9 & 0.93 & 093 & 0.033 & $\ldots$ \\
\hline 198.7 & 27.5 & 1.34 & 173 & 0.049 & $\ldots$ \\
\hline 183.2 & 25.7 & 6.79 & 145 & 0.264 & SE \\
\hline 168.7 & 26.1 & 6.77 & 163 & 0.253 & $\ldots$ \\
\hline 148.0 & 25.0 & 4.75 & 123 & 0.190 & $\ldots$ \\
\hline 153.1 & 25.3 & 5.48 & 126 & 0.217 & $\ldots$ \\
\hline 129.8 & 28.6 & 4.91 & 135 & 0.172 & $\ldots$ \\
\hline 104.0 & 27.8 & 5.30 & 098 & 0.191 & $\mathrm{NE}$ \\
\hline 098.0 & 29.8 & 4.77 & 110 & 0.160 & $\ldots$ \\
\hline 073.5 & 26.1 & 9.09 & 095 & 0.348 & $\ldots$ \\
\hline 062.5 & 23.3 & 7.26 & 076 & 0.312 & $\ldots$ \\
\hline 048.6 & 24.8 & 6.81 & 066 & 0.274 & $\ldots$ \\
\hline 034.9 & 21.2 & 6.96 & 053 & 0.329 & $\ldots$ \\
\hline
\end{tabular}

Note. ${ }^{\text {a }}$ P.A. means the position angle, measured counterclockwise from north.

Table 3

Averaged ZSQ Motions Grouped by Quadrants

\begin{tabular}{lcc}
\hline \hline Region & $\begin{array}{c}\text { Average } \mu \\
\left(\mathrm{mas} \mathrm{yr}^{-1}\right)\end{array}$ & $\begin{array}{c}\text { Average Expansion Scale Factor } \\
\left(\text { mas yr }^{-1} \operatorname{arcsec}^{-1}\right.\end{array}$ \\
\hline$\left[\mathrm{N}_{\text {II }}\right]$ Bright NW & $6.2 \pm 1.2$ & $0.22 \pm 0.05$ \\
{$\left[\mathrm{~N}_{\text { II }}\right]$ Bright SW } & $8.0 \pm 2.0$ & $0.27 \pm 0.04$ \\
{$\left[\mathrm{~N}_{\text {II }}\right]$ Bright SE } & $6.1 \pm 0.7$ & $0.21 \pm 0.05$ \\
{$\left[\mathrm{~N}_{\text {II }}\right]$ Bright NE } & $7.9 \pm 1.4$ & $0.26 \pm 0.07$ \\
{$\left[\mathrm{~N}_{\text {II }}\right]$ Bright NW+SE } & $6.2 \pm 0.9$ & $0.22 \pm 0.05$ \\
{$\left[\mathrm{~N}_{\text {II }}\right]$ Bright NE+SW } & $8.0 \pm 1.6$ & $0.27 \pm 0.05$ \\
F502N Dark NW & $4.4 \pm 1.2$ & $0.19 \pm 0.04$ \\
F502N Dark SW & $4.3 \pm 1.1$ & $0.15 \pm 0.04$ \\
F502N Dark SE & $5.7 \pm 1.6$ & $0.22 \pm 0.06$ \\
F502N Dark NE & $4.1 \pm 1.1$ & $0.17 \pm 0.05$ \\
F502N Dark NW+SE & $4.9 \pm 1.4$ & $0.20 \pm 0.05$ \\
F502N Dark NE+SW & $4.2 \pm 1.1$ & $0.16 \pm 0.04$ \\
\hline
\end{tabular}

indicates similarity of properties. This justifies grouping the results into the quadrants along the major $(\mathrm{NE}+\mathrm{SW})$ and minor $(\mathrm{NW}+\mathrm{SE})$ axes. We adopt $\langle E([\mathrm{~N}$ II $]$, minor $)\rangle=0.22 \pm 0.04$, $\langle E([\mathrm{~N}$ II $]$, major $)\rangle=0.27 \pm 0.05,\langle E($ F502N, minor $)\rangle=0.20 \pm$ 0.03 , and $\langle E(\mathrm{~F} 502 \mathrm{~N}$, major $)\rangle=0.18 \pm 0.02$ as the average of the ZSQ and magnification methods.

Our measurements of $\mu$ are limited to the Main Ring of the nebula and include no measurements of inner or outer
Table 4

Magnification Method Results for [N II] Bright Samples

\begin{tabular}{lrcc}
\hline \hline Sample & Optimum Magnifier & $\begin{array}{c}E \\
\left(\mathrm{mas} \mathrm{yr}^{-1} \operatorname{arcsec}\right)\end{array}$ & Quadrant \\
\hline $\mathrm{a}$ & $1.00275 \pm 0.00025$ & $0.21 \pm 0.02$ & $\mathrm{NW}$ \\
$\mathrm{b}$ & $1.0035 \pm 0.00025$ & $0.27 \pm 0.02$ & $\mathrm{NW}$ \\
$\mathrm{c}$ & $1.00288 \pm 0.00025$ & $0.22 \pm 0.02$ & $\mathrm{NW}$ \\
$\mathrm{d}$ & $1.00275 \pm 0.00025$ & $0.21 \pm 0.02$ & $\mathrm{SW}$ \\
$\mathrm{e}$ & $1.00425 \pm 0.00025$ & $0.33 \pm 0.02$ & $\mathrm{SW}$ \\
$\mathrm{f}$ & $1.0025 \pm 0.00025$ & $0.19 \pm 0.02$ & $\mathrm{SE}$ \\
$\mathrm{g}$ & $1.00325 \pm 0.00025$ & $0.25 \pm 0.02$ & $\mathrm{SE}$ \\
$\mathrm{h}$ & $1.0025 \pm 0.00025$ & $0.19 \pm 0.02$ & $\mathrm{SE}$ \\
$\mathrm{i}$ & $1.00425 \pm 0.00025$ & $0.33 \pm 0.02$ & $\mathrm{NE}$ \\
$\mathrm{j}$ & $1.00325 \pm 0.00025$ & $0.25 \pm 0.02$ & $\mathrm{NE}$ \\
\hline
\end{tabular}

Note. Sample regions are shown in Figure 3.

halo features. O'Dell et al. (2007) first established and O'Dell et al. (2013a) confirmed the ellipticity of the Main Ring. The nebula's equatorial concentration of material is elliptical in form rather than the apparent ellipticity being the result of a circular ring seen at an angle. The small difference of the major and minor axis $\left\langle E\left(\left[\mathrm{~N}_{\mathrm{II}}\right]\right)\right\rangle$ values is consistent with the intrinsically elliptical equatorial concentration of the Main Ring. The minor axis material is expanding at a slower rate either because it was 
Table 5

Magnification Method Results for F502N Samples

\begin{tabular}{lrcc}
\hline \hline Sample & Optimum Magnifier & $\begin{array}{c}E \\
\left(\mathrm{mas} \mathrm{yr}^{-1} \text { arcsec }\right)\end{array}$ & Quadrant \\
\hline A & $1.0025 \pm 0.00025$ & $0.19 \pm 0.02$ & NW \\
B & $1.00275 \pm 0.00025$ & $0.21 \pm 0.02$ & NW \\
C & $1.00225 \pm 0.00025$ & $0.17 \pm 0.02$ & NW \\
D & $1.00225 \pm 0.00025$ & $0.17 \pm 0.02$ & SW \\
E & $1.00225 \pm 0.00025$ & $0.17 \pm 0.02$ & SW \\
F & $1.0025 \pm 0.00025$ & $0.19 \pm 0.02$ & SW \\
G & $1.00275 \pm 0.00025$ & $0.21 \pm 0.02$ & SE \\
H & $1.00275 \pm 0.00025$ & $0.21 \pm 0.02$ & $\mathrm{NE}$ \\
I & $1.0025 \pm 0.00025$ & $0.19 \pm 0.02$ & $\mathrm{NE}$ \\
J & $1.00225 \pm 0.00025$ & $0.17 \pm 0.02$ & $\mathrm{NE}$ \\
\hline
\end{tabular}

Note. Sample regions are shown in Figure 3.

subject to less force during the phase when the Main Ring was being formed, or the overburden of outer neutral material is greater there.

\subsection{Relative Velocities of the Dark Knots and the Nebular Gas}

Systematic differences of the averaged $E$ values in [N II] and F502N are seen to exist along both axes. This difference is greatest along the major axis, where $\left\langle E\left(\left[\mathrm{~N}_{\mathrm{II}}\right]\right)\right\rangle=0.27 \pm 0.05$ and $\langle E(\mathrm{~F} 502 \mathrm{~N})\rangle=0.18 \pm 0.02$. The difference along the minor axis is much more uncertain, with $\left\langle E\left(\left[\mathrm{~N}_{\mathrm{II}}\right]\right)\right\rangle=0.22 \pm 0.04$ and $\langle E(\mathrm{~F} 502 \mathrm{~N})\rangle=0.20 \pm 0.03$. A smaller value of $E(\mathrm{~F} 502 \mathrm{~N}$, dark knots) as compared with $E$ ([N II], bright gas) indicates that the dark knots do not expand as fast as the ionized gas in which they exist.

Two families of models are used to explain the origin of the dark knots found in PNe. The first (Dyson et al. 1989) argues that the knots are condensations of dust and gas that originated in the extended atmosphere of the central star while it was still at the top of the asymptotic giant branch. The similarity of the $E$ values found today argue against this origin. It would be highly unlikely that knots sufficiently dense to survive the first phases of shell ejection would receive a radial acceleration this similar to that of the low-density gas. The second set of models (Capriotti 1973; Vishniac 1983; García-Segura et al. 2006) invokes formation of the knots much later in the life of the nebula. Considerable observational evidence (O'Dell et al. 2002, 2004) indicates that the knots arise near the main ionization front. Our results show that the knots, once formed, appear to lag behind the motion of the surrounding gas, and this feature must be factored into theories of their formation and calculations of the subsequent properties.

Previously, Meaburn et al. (1998) argued that the system of well-defined dark knots in the Helix Nebula is expanding at a rate of about one-half that of the nebular gas. This conclusion was drawn from the study of the radial velocities of the small crescents of ionized gas formed on the side of the knots facing the central star. The primary concentration of gas in the Helix Nebula is in an equatorial ring lying almost in the plane of the sky (Meaburn et al. 1998; O'Dell et al. 2004), much like the Ring Nebula. Expansion motions are nearly in the plane of the sky, meaning that results determined from radial velocities are inherently less valuable. A study of the tangential velocities should be more accurate for addressing the question of the relative motion of the knots. However, it appears that the study of tangential velocities of the Ring Nebula and the radial velocities
Table 6

Averaged Magnification Method Results Grouped by Quadrants

\begin{tabular}{lcc}
\hline \hline Region & $\begin{array}{c}\text { 1-Average Optimum } \\
\text { Magnifier }\end{array}$ & $\begin{array}{c}\text { Average Expansion } \\
\text { Scale Factor } \\
\left(\text { mas yr }^{-1} \text { arcsec }\right)\end{array}$ \\
\hline$\left[\mathrm{N}_{\text {II }}\right]$ Bright NW & $3.04 \pm 0.40$ & $0.23 \pm 0.03$ \\
{$[\mathrm{~N}$ II $]$ Bright SW } & $3.50 \pm 0.70$ & $0.27 \pm 0.06$ \\
{$[\mathrm{~N}$ II $]$ Bright SE } & $2.80 \pm 0.35$ & $0.21 \pm 0.04$ \\
{$[\mathrm{~N}$ II] Bright NE } & $3.75 \pm 0.5$ & $0.29 \pm 0.04$ \\
{$[\mathrm{~N}$ II] Bright NW+SE } & $2.73 \pm 0.30$ & $0.22 \pm 0.03$ \\
{$[\mathrm{~N}$ II] Bright NE+SW } & $3.62 \pm 0.75$ & $0.28 \pm 0.06$ \\
F502N Dark NW & $2.50 \pm 0.25$ & $0.19 \pm 0.02$ \\
F502N Dark SW & $2.33 \pm 0.14$ & $0.18 \pm 0.01$ \\
F502N Dark SE & $2.75 \pm 0.25$ & $0.21 \pm 0.02$ \\
F502N Dark NE & $2.5 \pm 0.25$ & $0.19 \pm 0.02$ \\
F502N Dark NW+SE & $2.60 \pm 0.22$ & $0.20 \pm 0.02$ \\
F502N Dark NE+SW & $2.42 \pm 0.20$ & $0.18 \pm 0.02$ \\
\hline
\end{tabular}

in the Helix Nebula come to the same conclusion: the knots are expanding more slowly and are decoupled from the gas motion.

\subsection{The Distance to the Ring Nebula}

A distance $(D)$ for the Ring Nebula can be obtained from an accurate knowledge of its dynamical properties. For homologous expansion, the relation that applies is $D(\mathrm{pc})=$ $\left(k_{1} \times \mathcal{R} \times \cos \theta\right) /(4.74 E)$, where $k_{1}$ is the scale factor relating the spatial expansion velocity $\left(\mathrm{km} \mathrm{s}^{-1}\right)$ to the true (not projected) distance to the central star (expressed as "), $\mathcal{R}$ is the pattern velocity (the ratio of the ionization front velocity compared with the gas velocity), $\theta$ is the angle of the equatorial plane of the nebula to the sky $\left({ }^{\circ}\right)$, and $E$ is the expansion scale ratio. Adopting the O'Dell et al. (2007) values of $k_{1}=0.65\left(\mathrm{~km} \mathrm{~s}^{-1}\right.$ arcsec) and $\mathcal{R}=1.34$, the relation becomes $D(\mathrm{pc})=179 \times E^{-1}$, where $E$ is in mas $\mathrm{yr}^{-1}$ arcsec. From this work we adopt the average of the major and minor axes for $[\mathrm{N}$ II $]\left(E=0.25 \mathrm{mas} \mathrm{yr}^{-1}\right.$ arcsec $)$ and find $D=716 \mathrm{pc}$. It is difficult to identify an uncertainty in this distance because it is dependent upon the validity of the dynamical model used. The key uncertainties are in the determination of $k_{1}$ (O'Dell et al. 2007) and $\mathcal{R}$ (O'Dell et al. 2009). The resulting uncertainty in the distance is probably about $30 \%$. The derived distance should be compared with the astrometric distance of $700 \pm_{200}^{450} \mathrm{pc}$ (Harris et al. 2007). Our rounded value of $720 \mathrm{pc}$ is the best distance to be adopted. This is only a small change from the previously recommended value of $740 \mathrm{pc}$ (O’Dell et al. 2009).

\subsection{Timescales}

The reciprocal of the expansion ratio has the dimension of years and represents the time since the onset of the assumed homologous expansion. Adopting the average of $E=0.25$ (Section 3.3) gives $4000 \mathrm{yr}$ since the onset of the expansion. This method of calculation produces an overly simplified dynamical age and only has the meaning as a measure of when the basic dynamical properties of the expanding nebular shell were established under the assumption that the size at that point was small.

The current characteristics of the central star are $\left(\log L / L_{\odot}\right)=$ $2.3 \pm 0.2$ and $\log T \approx 5.1 \pm 0.02$ ) (O’Dell et al. 2007) and comparison with the theoretical path for a collapsing star of about $M=0.62 M_{\odot}$ (Blöcker 1995) indicates that the time since the onset of stellar collapse is about $5000 \mathrm{yr}$, i.e., similar to the dynamic age. 


\section{SUMMARY AND CONCLUSIONS}

We have been able to utilize early HST/WFPC2 images of the Ring Nebula in combination with recent WFC3 images to determine tangential motions of [ $\mathrm{N}$ II] emission features near the main ionization front of this ionization-bounded nebula. We could also determine the tangential velocities of the dark knots seen as extinction of the diffuse nebular [O III] emission.

The well-defined radial expansion patterns were found in both the $\left[\mathrm{N}_{\mathrm{II}}\right]$ emission-line features and the dark knots. The results from a second method of determination of the motions under the assumption of homologous expansion are in excellent agreement with the results from measurement of individual features.

The expansion scale ratio $E$ that characterizes the scale of motions within the nebula is not the same on both axes of the Main Ring. It is larger for the major axis, a result consistent with the inherent ellipticity of the Main Ring. The apparent ellipticity of the Main Ring is primarily due to its inherent shape rather than being the result of a circular object observed at an angle to the plane of the sky. The suggestions of spiral structure are the results of the asymmetries of the boundaries of the Main Ring and the lobes (O'Dell et al. 2013a).

The reciprocal of the expansion scale factor indicates that the dynamic age of the Ring Nebula is about 4000 yr. Combining the new results for the expansion factor and the previously established spatial expansion velocity scale factor determined from radial velocities has allowed the calculation of a distance to the Ring Nebula of 720 pc, with a likely uncertainty of $30 \%$.

We are grateful to David Thompson of the Large Binocular Telescope Observatory for providing copies of his unpublished LBTO $\mathrm{H}_{2}$ data taken with the LUCI1 instrument and to Zoltan Levay of the Space Telescope Science Institute for a fruitful discussion on this study.

G.J.F. acknowledges support by NSF (0908877, 1108928, and 1109061), NASA (10-ATP10-0053, 10-ADAP10-0073, and NNX12AH73G), JPL (RSA No. 1430426), and STScI (HST-AR-12125.01, GO-12560, and HST-GO-12309). M.P. received partial support from CONACyT grant 129553. W.J.H. acknowledges financial support from DGAPA-UNAM through project PAPIIT IN102012. C.R.O.'s participation was supported in part by HST programs GO 12309 and GO 12543.

Facility: HST (WFC3)

\section{REFERENCES}

Blöcker, T. 1995, A\&A, 299, 755

Capriotti, E. R. 1973, ApJ, 179, 745

Currie, D. G., Dowling, D. M., Shaya, E. J., et al. 1996, AJ, 112, 1115

Dyson, J. E., Hartquist, T. W., Pettini, M., \& Smith, L. J. 1989, MNRAS, 241,625

García-Segura, G., López, J. A., Steffen, W., Meaburn, J., \& Manchado, A. 2006, ApJL, 646, L61

Gómez, Y., Rodriguez, L. F., \& Moran, J. M. 1993, ApJ, 416, 620

Hajian, A. R., Terzian, Y., \& Bignell, C. 1993, AJ, 106, 1965

Hajian, A. R., Terzian, Y., \& Bignell, C. 1995, AJ, 109, 2600

Harris, H. C., Dahn, C. C., Canzian, B., et al. 2007, AJ, 133, 631

Hartigan, P., Morse, J. A., Reipurth, B., Heathcote, S., \& Bally, J. 2001, ApJL, 559, L157

Liller, M. H., Welther, B. L., \& Liller, W. 1966, ApJ, 144, 280

Masson, C. R. 1986, ApJL, 302, L27

Meaburn, J., Clayton, C. A., Bryce, M., et al. 1998, MNRAS, 294, 201

O’Dell, C. R., Balick, B., Hajian, A. R., Henney, W. J., \& Burkert, A. 2002, AJ, 123,3329

O’Dell, C. R., Ferland, G. J., Henney, W. J., \& Peimbert, M. 2013a, AJ, 145,92

O’Dell, C. R., Ferland, G. J., Henney, W. J., \& Peimbert, M. 2013b, AJ, 145, 93

O'Dell, C. R., Henney, W. J., \& Ferland, G. J. 2005, AJ, 130, 172

O’Dell, C. R., Henney, W. J., \& Sabbadin, F. 2009, AJ, 137, 3815

O’Dell, C. R., McCullough, P. R., \& Meixner, M. 2004, AJ, 128, 2339

O’Dell, C. R., Sabbadin, F., \& Henney, W. J. 2007, AJ, 134, 1679

Palen, S., Balick, B., Hajian, A. R., et al. 2002, AJ, 123, 2666

Reed, D. S., Balick, B., Hajian, A. R., et al. 1999, AJ, 118, 2430

Terzian, Y. 1997, in Planetary Nebulae, ed. H. J. Habing \& H. J. G. L. M. Lamers (Dordrecht: Kluwer), 29

Vishniac, E. T. 1983, ApJ, 274, 152

Wilson, O. C. 1950, ApJ, 111, 279 